\title{
NOT EVERY NEAR FAMILY IS CONTAINED IN A CLAN
}

\author{
S. A. NAIMPALLY AND J. H. M. WHITFIELD
}

ABSTRACT. In this note we solve an open problem concerning near structures and show that, unlike proximities and contiguities, not every near structure is induced by an elementary one.

Recently Thron [4] has shown that if $\Pi$ is a basic proximity and $A \epsilon$ $\Pi(B)$, then there is a $\Pi$-clan $\sigma$ which contains both $A$ and $B$. It is easy to extend this result to contiguities. In this paper we show by means of an example ${ }^{1}$ that an analogous result does not hold for near structures (see [2], [3] for further references). Our example also shows that a near family need not be contained in a maximal near family (see also [3]).

Let $R$ denote the space of real numbers with the usual topology and let $m^{*}$ denote the Lebesgue outer measure. Define $\lambda$ by $\mathbb{A} \in \lambda$ iff $\bigcap\left\{A^{-}\right.$: $A \in \mathbb{Q}\} \neq \varnothing$ or $\operatorname{Inf}\left\{m^{*}\left(A^{-}\right): A \in \mathbb{Q}\right\}>0$. It is easily verified that $\lambda$ is a compatible $L O$-near structure on $R$. Clearly if $A_{n}=[n-1 / 10, n+1 / 10]$, then $\mathbb{Q}=\left\{A_{n}: n \in \mathbf{N}\right\} \in \lambda$. We now show that $\mathscr{Q}$ is not contained in any $\lambda$ clan. Suppose on the contrary, that $\mathbb{Q}$ is contained in a $\lambda$-clan $\sigma$. Then for each $n \in \mathrm{N}$ there is an ultrafilter $\mathcal{U}_{n}$ on $R$ with $A_{n} \in \mathcal{U}_{n} \subset \sigma$. However, for each $n \in \mathbf{N}$, there is a $B_{n} \in \mathcal{U}_{n}^{n}$ with $m^{*}\left(B_{n}^{-}\right)<1 / 10 n$. Clearly $B=\left\{B_{n}: n \in \mathbf{N}\right\} \notin \lambda$ which contradicts the fact that $\sigma$ is a clan.

If $(X, \lambda)$ is a separated $L O$-near space, then by considering the space of all $\lambda$-bunches and following a method analogous to one in [1], one can prove the following result:

Theorem. Every $\mathcal{U} \in \lambda$ is contained in a $\lambda$-clan $\sigma$ if and only if $X$ can be densely embedded in a space $X^{*}$ such that $\lambda$ is the subspace nearness induced by the associated elementary nearness $\lambda_{0}$ on $X^{*}$.

Received by the editors November 19, 1973.

AMS (MOS) subject classifications (1970). Primary 54E05.

Key words and phrases. Nearness, near structures, clan, proximity, contiguity.

${ }^{1}$ Professor H. L. Bentley has pointed out to the authors that another counterexample was constructed in the Spring of 1973 by H. L. Bentley, W. N. Hunsaker, S. A. Naimpally and P. L. Sharma. This appears in his paper Nearness spaces and extensions of topological spaces. 
Our counterexample shows that not every near structure can be so simply obtained.

\section{REFERENCES}

1. M. S. Gagrat and S. A. Naimpally, Proximity approach to extension problems, Fund. Math. 71 (1971), 63-76. MR 45 \#2653.

2. M. S. Gagrat and W. J. Thron, Nearness and proximity extensions, Trans. Amer. Math. Soc. (to appear).

3. S. A. Naimpally, Reflective functors via nearness, Fund. Math. 85 (1974).

4. W. J. Thron, On a problem of $F$. Riesz concerning proximity structures, Proc. Amer. Math. Soc. 40 (1973), 323-326.

DEPARTMENT OF MATHEMATICAL SCIENCES, LAKEHEAD UNIVERSITY, THUNDER BAY, ONTARIO P7B SE1, CANADA 